\title{
Dissecting the Wolf-Hirschhorn syndrome phenotype: WHSC1 is a neurodevelopmental gene contributing to growth delay, intellectual disability, and to the facial dysmorphism
}

\author{
Marcella Zollino $^{1} \cdot$ Paolo Niccolo' Doronzio ${ }^{1}$
}

Received: 15 May 2018 / Revised: 15 May 2018 / Accepted: 17 May 2018 / Published online: 8 June 2018

(c) The Author(s) under exclusive licence to The Japan Society of Human Genetics 2018

We read with great interest the report by Konovalov et al. in the present issue of the journal, dealing with a de novo stop gain mutation in WHSC1, which was detected by wholeexome sequencing (WES) in association with growth delay, some facial features resembling the Wolf-Hirschhorn syndrome (WHS) phenotype, and intellectual disability (ID). WHSCl is one of the major pathogenic genes for WHS. However, intragenic mutations in this gene were never reported before.

\section{The core WHS phenotype and the WHS critical region}

WHS (OMIM 194190) is a contiguous gene syndrome caused by partial $4 \mathrm{p}$ deletion.

Following its first description in 1965, in association with large chromosome deletions, nosology of WHS in the 1960s and 1980s points out a severe phenotype, with clinical manifestations including severe ID, severe growth delay, the famous facial "Greek warrior helmet" profile, seizures, and major malformations, including congenital heart defects, midline defects, renal abnormalities, and skeletal anomalies [1].

However, nosology of WHS has changed with time. The subsequent techniques of molecular cytogenetics, in fact, have allowed for the diagnosis of small deletions that are usually associated with a milder phenotype lacking major malformations. Accordingly, two categories of the WHS

Marcella Zollino

marcella.zollino@unicatt.it

1 Institute of Genomic Medicine, Catholic University, A. Gemelli Foundation, Rome, Italy phenotype were distinguished, which were referred to as classical and mild, respectively [2].

It must be specified that, associated with certain very small or atypical $4 \mathrm{p}$ deletion, the diagnosis of WHS can be questionable in some cases, prompting clinical geneticists to establish strict clinical diagnostic criteria for WHS. In front of the great variability of the WHS phenotype, depending mostly on the extent of the $4 p$ deletion, there is a consensus in considering the core WHS phenotype as defined by the association of ID, growth delay, the typical facial dysmorphism, and seizures (or electroencephalographic anomalies) [2,3]. All these signs are regarded as minimal diagnostic criteria for WHS, and they all map within the 1.9 $\mathrm{Mb}$ terminal region on $4 \mathrm{p}$.

\section{The WHS critical region and candidate genes}

As a role, the critical genomic region for WHS would be the smallest region whose haploinsufficiency gives rise to the core phenotype. The first critical region, WHSCR, was described in 1997 [4], limited to a $165 \mathrm{~kb}$ interval at about 2 $\mathrm{Mb}$ from the $4 \mathrm{p}$ telomere. Two genes, WHSCl (OMIM 602952), two thirds of which map within the distal half of the WHSCR, and WHSC2 (OMIM 606026), falling entirely within WHSCR, were described as candidate genes. It was tentatively suggested that a single gene can be responsible for the whole WHS phenotype, acting as master regulator of transcription [4]

A unique patient was reported by Rauch et al. [5]. with a small interstitial deletion restricted to WHSCR. As pointed out by the authors, this patient presented with an atypical WHS phenotype, with no full WHS facial dysmorphism, thin habitus, but normal height, and, importantly, with no seizures.

The detection of a $1.9 \mathrm{Mb}$ deletion productive of the full WHS core phenotype, including seizures, but extending 
beyond WHSCR, allowed re-definition of the critical region for WHS. The newly described region was referred to as WHSCR-2 [6]. WHSCR-2 lies contiguous, distally, to WHSCR, sharing with it WHSC1, but including, in addition, LETM1 (OMIM 604407).

\section{Pathogenesis of the WHS phenotype and candidate genes}

It must be specified that, after the initial and very transient hypothesis that a single gene can cause the whole WHS phenotype, pathogenesis of WHS is largely considered to be multigenic.

In particular, LETMI (leucine zipper/EF-hand containing transmembrane, OMIM 604407), which is involved in $\mathrm{Ca} 2$ signaling, is the canonical candidate gene for seizures, and WHSC1 (OMIM *602952, coding for a H3K36me3specific histone methyltransferase) is the major candidate gene for growth delay and the typical facial dysmorphism.

However, there is definite evidence that haploinsufficiency of LETM1 alone is not sufficient in causing the WHS-associated seizure disorder. Synergic haploinsufficiency of additional genes is requested, according to a comorbidity model of pathogenesis, most likely. New candidate genes for seizures are CPLX1 (OMIM 605032), encoding for a presynaptic regulatory protein [7] and $P I G G$ (OMIM 616918), involved in biosynthesis and transport of glycosylphosphatidylinositol-anchored proteins [8].

Although WHSCl is currently considered the major candidate gene for growth delay and for the facial dysmorphisms, synergic haploinsufficiency of additional genes, distal to it, is the likely mechanism for the full WHS facial phenotype as well [2, 3].

According to these considerations, the WHS critical region should be extended beyond both WHSCR and WHSCR-2, to encompass the interstitial $1.5 \mathrm{Mb}$ region between $1.9 \mathrm{Mb}$ to $0.4 \mathrm{Mb}$ from the $4 \mathrm{p}$ telomere [7].

Deciphering the major pathogenic genes in WHS can allow search for mechanisms and for molecularly based therapy. The current available techniques for whole genomic investigations, including chromosomal microarray and WES, have the extraordinary possibility to understand the role of either the canonical or any new candidate genes for the WHS pathogenesis.

\section{First report of loss-of-function mutation in WHSC1}

We are involved in the study of WHS since several years. Extensive genotype-phenotype correlations, including personal observations and analysis of literature reports, led us to infer that haploinsufficiency limited to WHSCl is expected to cause growth delay, several WHS facial features but not the full WHS facial dysmorphism, no seizures, no microcephaly, and no major malformations. Whether ID can be another component manifestation was unclear till now. The excellent report by Konovalov et al. fills the gap on the clinical outcome of WHSCl haploinsufficiency. The de novo stop gain mutation in WHSC1 was detected by WES, in absence of a clinical suspicion of WHS, in a subject who presented with growth delay, several WHS facial features but not the full WHS facial dysmorphism, no seizures, no microcephaly, no major malformations, and ID.

Some important considerations are in order.

First, haploinsufficiency of WHSCl is not sufficient to make a diagnosis of WHS. This particularly implies that no increased risk for seizures is expected. To avoid speaking in term of WHS is strongly recommended.

Then, WHSC1 appears to be a developmental gene causing impairment of both morphogenesis and neurodevelopment. It is to be considered a new autosomal gene responsible for syndromic ID, with physical manifestations overlapping in part with WHS but not featuring the typical WHS phenotype.

Finally, this report adds evidence about the multigenic pathogenesis of the core WHS phenotype.

\section{Compliance with ethical standards}

Conflict of interest The authors declare that they have no conflict of interest.

\section{References}

1. Wilson MG, Towner JW, Coffin GS, Ebbin AJ, Siris E, Brager P. Genetic and clinical studies in 13 patients with the WolfHirschhorn syndrome [del(4p)]. Hum Genet. 1981;59:297-307.

2. Zollino M, Murdolo M, Marangi G, Pecile V, Galasso C, Mazzanti $\mathrm{L}$, et al. On the nosology and pathogenesis of Wolf-Hirschhorn syndrome: genotype-phenotype correlation analysis of 80 patients and literature review. Am J Med Genet. 2008;148C:257-69. https:// doi.org/10.1002/ajmg.c.30190.

3. Battaglia A, Carey JC, South ST. Wolf-Hirschhorn syndrome: a review and update. Am J Med Genet C Semin Med Genet. 2015;169:216-23. https://doi.org/10.1002/ajmg.c.31449.

4. Wright TJ, Ricke DO, Denison K, Abmayr S, Cotter PD, Hirschhorn $\mathrm{K}$, et al. A transcript map of the newly defined $165 \mathrm{~kb}$ WolfHirschhorn syndrome critical region. Hum Mol Genet. 1997;6:317-24.

5. Rauch A, Schellmoser S, Kraus C, Dörr HG, Trautmann U, Altherr MR, et al. First known microdeletion within the Wolf-Hirschhorn syndrome critical region refines genotype-phenotype correlation. Am J Med Genet. 2001;99:338-42.

6. Zollino M, Lecce R, Fischetto R, Murdolo M, Faravelli F, Selicorni A, et al. Mapping the Wolf-Hirschhorn syndrome phenotype outside the currently accepted WHS critical region and defining a new critical region, WHSCR-2. Am J Hum Genet. 2003;72:590-7. https://doi.org/10.1086/367925. 
7. Zollino M, Orteschi D, Ruiter M, Pfundt R, Steindl K, Cafiero C, et al. Unusual 4p16.3 deletions suggest an additional chromosome region for the Wolf-Hirschhorn syndrome-associated seizures disorder. Epilepsia. 2014;55:849-57. https://doi.org/10.1111/ epi.12617.
8. Ho KS, South ST, Lortz A, Hensel CH, Sdano MR, Vanzo RJ, et al. Chromosomal microarray testing identifies a $4 p$ terminal region associated with seizures in Wolf-Hirschhorn syndrome. J Med Genet. 2016;53:256-63. https://doi.org/10.1136/jmedgenet-2015103626. 